\title{
The structural equation analysis of childhood abuse, adult stressful life events, and temperaments in major depressive disorders and their influence on refractoriness
}

\author{
This article was published in the following Dove Press journal: \\ Neuropsychiatric Disease and Treatment \\ 14 August 2015 \\ Number of times this article has been viewed
}

\section{Hiroyuki Toda,' Takeshi Inoue, ${ }^{2,3}$ Tomoya Tsunoda,' Yukiei Nakai, ${ }^{2}$ Masaaki Tanichi,' Teppei Tanaka,' Naoki Hashimoto, ${ }^{2}$ Yasuya Nakato, ${ }^{2}$ Shin Nakagawa, ${ }^{2}$ Yuji Kitaichi, ${ }^{2}$ Nobuyuki Mitsui, ${ }^{2}$ Shuken Boku, ${ }^{4}$ Hajime Tanabe, ${ }^{5}$ Masashi Nibuya, ' Aihide Yoshino, ' Ichiro Kusumi ${ }^{2}$}

'Department of Psychiatry, National Defense Medical College, Tokorozawa, Saitama, Japan; ${ }^{2}$ Department of Psychiatry, Hokkaido University Graduate School of Medicine, Sapporo, Japan; ${ }^{3}$ Department of Psychiatry, Tokyo Medical University,

Tokyo, Japan; ${ }^{4}$ Department of Psychiatry, Kobe University Graduate School of Medicine, Kobe, Japan; ${ }^{5}$ Department of Clinical Human Sciences, Graduate School of Humanities and Social Sciences, Shizuoka University, Shizuoka, Japan

Correspondence: Hiroyuki Toda Department of Psychiatry, National Defense Medical College, Namiki 3-2, Tokorozawa, Saitama 359-85I3, Japan Tel +8I 42995 I6I9

Fax +8I 429965203

Email toda1973@ndmc.ac.jp

Takeshi Inoue

Department of Psychiatry, Tokyo Medical University, 6-7-I, Nishishinjuku, Shinjuku-ku, Tokyo, 160-0023, Japan

$\mathrm{Tel}+8$ I 333426 III

Fax +8 I 333404499

Email tinoue@tokyo-med.ac.jp
Background: Previous studies have shown the interaction between heredity and childhood stress or life events on the pathogenesis of a major depressive disorder (MDD). In this study, we tested our hypothesis that childhood abuse, affective temperaments, and adult stressful life events interact and influence the diagnosis of MDD.

Patients and methods: A total of 170 healthy controls and 98 MDD patients were studied using the following self-administered questionnaire surveys: the Patient Health Questionnaire-9 (PHQ-9), the Life Experiences Survey, the Temperament Evaluation of the Memphis, Pisa, Paris, and San Diego Autoquestionnaire, and the Child Abuse and Trauma Scale (CATS). The data were analyzed with univariate analysis, multivariable analysis, and structural equation modeling.

Results: The neglect scores of the CATS indirectly predicted the diagnosis of MDD through cyclothymic and anxious temperament scores of the Temperament Evaluation of the Memphis, Pisa, Paris, and San Diego Autoquestionnaire in the structural equation modeling. Two temperaments - cyclothymic and anxious - directly predicted the diagnosis of MDD. The validity of this result was supported by the results of the stepwise multivariate logistic regression analysis as follows: three factors - neglect, cyclothymic, and anxious temperaments - were significant predictors of MDD. Neglect and the total CATS scores were also predictors of remission vs treatment-resistance in MDD patients independently of depressive symptoms.

Limitations: The sample size was small for the comparison between the remission and treatment-resistant groups in MDD patients in multivariable analysis.

Conclusion: This study suggests that childhood abuse, especially neglect, indirectly predicted the diagnosis of MDD through increased affective temperaments. The important role as a mediator of affective temperaments in the effect of childhood abuse on MDD was suggested.

Keywords: neglect, major depressive disorder, affective temperament, mediator

\section{Introduction}

Various factors, such as genetic factors, environmental factors, and personality traits, are known to be risk factors for the development of a major depressive disorder (MDD). ${ }^{1-9}$ Unexpectedly, the heritability of MDD is not high (37\%) compared with schizophrenia (81\%) and bipolar disorder (85\%). ${ }^{10}$ Accordingly, other factors, such as environmental factors and personality traits, are presumed to contribute largely to the development of MDD. Adult stressful life events and childhood abuse are wellknown major environmental factors for MDD,,$^{3-5,11,12}$ and interestingly, these two 
environmental factors interact with genetic factors in the gene-by-environment $(G \times E)$ interaction to predict depressive symptoms and the onset of MDD, which may explain the relatively low heritability of MDD ${ }^{1,6}$ Moreover, the interaction between adult stressful life events and personality traits also facilitates the onset of MDD. ${ }^{7}$ However, no reports have been performed to show the association of the abovementioned three factors, ie, personality traits, adult stressful life events, and childhood abuse, by using structural equation modeling (SEM).

A meta-analysis revealed that childhood maltreatment was associated with a lack of response or remission during treatment for depression, ${ }^{13}$ and the included studies investigated depressed patients who had not responded to only one adequate antidepressant treatment, ie, stage 1 MDD according to the staging of depression based on prior treatment response proposed by Thase and Rush. ${ }^{14}$ On the other hand, Thase and Rush ${ }^{14}$ defined treatment-resistant depression (TRD) as treatment nonresponse (ie, the persistence of significant depressive symptoms) despite at least two treatment trials with drugs from different pharmacological classes, each used in an adequate dose for a sufficient period of time (stage 2 MDD-TRD). This definition is reasonable because clinical findings suggest that $20 \%-70 \%$ of nonresponders to antidepressants will respond to a different type of antidepressant. ${ }^{14,15}$ There have been only a few studies that investigate the demographic and clinical features in stage 2 MDD-TRD. ${ }^{16,17}$ No previous studies have investigated the environmental factors associated with stage 2 MDD-TRD. In association with personality traits, TRD demonstrated high scores for harm avoidance and low scores for reward dependence, self-directedness, and cooperativeness in the Temperament and Character Inventory. ${ }^{18}$ To the best of our knowledge, no previous studies have investigated affective temperament with stage 2 MDD-TRD.

Recently, we reported that childhood abuse indirectly increased the severity of depressive symptoms through increased affective temperaments and that affective temperaments directly increased the severity of depressive symptoms in the SEM of a nonclinical general adult population. ${ }^{9}$ Moreover, increased affective temperaments increased the negative appraisal of stressful life events, which, in turn, increased depressive symptoms mildly but significantly. ${ }^{9}$ This study was the first study to show the association of temperament, childhood abuse, and adult stressful life events with the depressive symptoms using the SEM in a nonclinical general population and the role of affective temperaments as mediators in the influence of childhood abuse on depressive symptoms. Such a relationship has not been reported in MDD patients, and whether this hypothesis can be extended to MDD populations should be verified.

The aim of this study is to clarify whether the effect and interaction of these three factors, personality traits, adult stressful life events, and childhood abuse, are involved in the distinction of MDD patients and healthy controls. Therefore, we compared affective temperaments identified on the Temperament Evaluation of the Memphis, Pisa, Paris, and San Diego Autoquestionnaire (TEMPS-A),${ }^{19}$ childhood abuse evaluated by the Child Abuse and Trauma Scale (CATS), ${ }^{20}$ adult stressful life events evaluated by the Life Experiences Survey (LES), ${ }^{21}$ and demographic and clinical features in MDD patients and healthy controls, and analyzed these interactions by SEM. Moreover, we studied whether affective temperaments, childhood abuse, and adult stressful life events are associated with stage 2 MDD-TRD.

\section{Materials and methods Subjects}

Healthy controls (control) included 170 subjects (103 male, 67 female) who did not fulfill the criteria of the Mini-International Neuropsychiatric Interview screen ${ }^{22}$ or did not fulfill the criteria of major or other depressive episodes as screened by the Patient Health Questionnaire-9 (PHQ-9). ${ }^{23}$ Control subjects were recruited previously and described in our previous study of the nonclinical general adult population. ${ }^{9}$

Ninety-eight patients (65 male, 33 female) with MDD had been treated at the Department of Psychiatry, National Defense Medical College; Hokkaido University Hospital; Self-Defense Forces Central Hospital; and Self-Defense Forces Sapporo Hospital. The patients were recruited from April 2012 to April 2013. The inclusion criteria were (a) meeting the criteria for a MDD in accordance with the DSM-IV-TR (Diagnostic and Statistical Manual of Mental Disorders, fourth edition, text revision) (American Psychiatric Association, 2000) ${ }^{24}$ by the psychiatrists responsible for these patients, (b) being 20 years of age or older, (c) not having severe physical diseases, (d) not meeting the diagnosis of axis II based on the DSM-IV-TR, (e) not having organic brain disease, and (f) having the capacity to consent to this study. Treatment-resistant major depression was defined as the persistence of significant or moderate depressive symptoms despite at least two treatment trials with antidepressants from different pharmacological classes (stage $2 \mathrm{MDD}$ according to the staging of depression based on prior treatment response proposed by Thase and Rush ${ }^{15}$ ). Each prior treatment must have been used in an adequate dose for an adequate period 
(ie, a minimum of the effective dose described in the product datasheet for 8 weeks). Remission was defined as patients who had no significant signs or symptoms of the disturbance during the past 2 months based on the DSM-IV-TR.

The authors investigated the demographic and psychosocial characteristics of age, sex, years of education, employment status, marital status, living alone or not, number of offspring, family history of a first-degree relative with a mood disorder, comorbid physical disease, psychiatric comorbidities, melancholic features based on the DSM$I V-T R$, number of previous depressive episodes, and illness duration from the first depressive episode in each patient. Six questionnaires, which are shown below, were distributed and self-completed. The 17-item Hamilton Depression Rating Scale (HDRS) and the Young Mania Rating Scale (YMRS) were administered to MDD patients but not to healthy controls. ${ }^{25,26}$

Written informed consent was obtained from all the subjects. The patients who did not have the capacity to consent were excluded in this study. This study was performed in accordance with the 1964 Declaration of Helsinki, revised in 2008, and was approved by the Institutional Review Board of the National Defense Medical College and Hokkaido University Hospital.

\section{Questionnaires}

\section{Patient Health Questionnaire-9}

The Japanese version of the PHQ-9 was self-completed by the subjects in the written form. ${ }^{27}$ This study employed a summary score for assessing the severity of depressive symptoms.

\section{Life Experiences Survey}

The Japanese version of the LES is a 57-item self-report measure that allows respondents to indicate events that they have experienced during the past year. ${ }^{9,21}$ The format of the LES calls for subjects to rate separately the desirability and effect of the events that they have experienced. They are asked to indicate the events experienced during the past year (0-6 months or 7 months-1 year) as well as (a) whether they viewed the event as being positive or negative and (b) the perceived impact of the particular event on their life at the time of occurrence. The ratings are on a 7-point scale ranging from extremely negative $(-3)$ to extremely positive $(+3)$. Summing the impact ratings of those events designated as positive by the subject provides a positive change score. A negative change score is derived by summing the impact ratings of those events experienced as negative by the subject.
Temperament Evaluation of the Memphis, Pisa, Paris, and San Diego Autoquestionnaire

The TEMPS-A is a self-rating questionnaire consisting of 109 items for men and 110 items for women. ${ }^{19}$ The subjects completed the Japanese standardized version of the TEMPS-A, which is a true $(=2)$ and false $(=1)$ questionnaire measuring the following temperament dimensions: depressive, cyclothymic, hyperthymic, irritable, and anxious. ${ }^{28}$

\section{Child Abuse and Trauma Scale}

The CATS is a 38-item scale. Initial findings have demonstrated that this measure has strong internal consistency (Cronbach's alpha $=0.63-0.90)$ and test-retest reliability $(r=0.71-0.91) .{ }^{20}$ The CATS has been shown to significantly correlate with outcome measures, such as dissociation, depression, stressful life events, and interpersonal difficulties. On each item, participants rate how frequently a particular abusive experience occurred to them during their childhood and adolescence, using a scale of $0-4$ ( $0=$ never; $4=$ always). The score for each subscale is the mean score on the items that make up that subscale. There are three subscales, measuring subjective reports of three aspects of adverse childhood experience: neglect/negative home atmospheres, punishment, and sexual abuse.

To confirm whether depressive symptoms influence CATS scores, CATS and PHQ-9 were measured twice in $50 \mathrm{MDD}$ patients at intervals of 1 month or longer. The total scores of the CATS were not significantly predicted by the PHQ-9 scores in a multiple regression analysis.

Hajime Tanabe, one of the authors, developed and validated the Japanese version of the CATS by the classic translation-back translation technique with the permission and confirmation of Dr Sanders, the developer of the CATS. $^{29}$

\section{State-Trait Anxiety Inventory, Form Y}

The Spielberger State-Trait Anxiety Inventory, Form Y $(\mathrm{STAI}-\mathrm{Y})^{30}$ is a 40 -item self-report measure of enduring (trait) and transient (state) anxiety symptoms. Respondents evaluate how they feel generally or at a particular moment on a 4-point scale in each statement. The STAI-Y state and trait scores range from 20 to 80 . The subjects completed the Japanese adaptation version of the STAI-Y (STAI-JYZ). ${ }^{31}$ State anxiety is designed to evaluate a temporary condition experienced in specific situations, "how respondents feel right now, at this moment", while trait anxiety is designed to evaluate a general tendency to perceive situations as threatening, "how respondents feel generally". 


\section{Data analysis}

We designed a structural equation model, in which MDD was predicted by childhood abuse, temperaments, and adult stressful life events. The models were assessed using multiple adequacies of fit criteria: the $\chi^{2}$, the comparative fit index (CFI), the Tucker-Lewis index (TLI), and the root mean square error of approximation (RMSEA). A non-significant $\chi^{2}(P>0.05)$ is suggestive of a good match between the data and the hypothesized model. According to the conventional criteria, a CFI greater than 0.95 , a TLI greater than 0.95 , and an RMSEA less than 0.08 indicate an acceptable fit and a CFI greater than 0.97, a TLI greater than 0.97 , and an RMSEA less than 0.05 indicate a good fit. ${ }^{32}$

The demographic and clinical characteristics and psychological measurement values were compared between the two groups using the Mann-Whitney $U$-test for continuous data and the $\chi^{2}$ test or, when appropriate, the Fisher's exact test for categorical data and were compared between the three groups by the Kruskal-Wallis test, followed by the Steel-Dwass post hoc test. Multivariate logistic regression analysis and multiple regression analysis were used to determine the correlation between the parameters and the predictive factors.

The statistical analyses were conducted using Mplus version 7.11 (Muthén \& Muthén, Los Angeles, CA, USA) for the SEM; SPSS Statistics version 21 (SPSS Inc., Chicago, IL, USA) for the multiple logistic regression analyses, the multiple regression analyses, $\chi^{2}$ test, the Mann-Whitney $U$-test, and the unpaired $t$-test; and the EZR ${ }^{33}$ (Saitama Medical Center, Jichi Medical University, Saitama, Japan) for the Kruskal-Wallis test, which is a graphical user interface for R (The R Foundation for Statistical Computing, Vienna, Austria). The differences were considered to be statistically significant at $P<0.05$. We did not apply Bonferroni correction to many variables because of the relatively small sample size of patients in this study.

\section{Results}

\section{Demographic and clinical characteristics} and the PHQ-9, CATS, TEMPS-A, and LES scores of the control and MDD groups

The demographic and clinical characteristics and the PHQ-9, CATS, TEMPS, and LES scores of 170 control and 98 MDD subjects are presented in Table 1. The duration of education; the number of offspring; the PHQ-9 summary scores; the sexual, neglect, and total scores of the CATS; the depressive, cyclothymic, irritable, and anxious temperament scores of the
TEMPS-A; and the negative change score of the LES were significantly different between the control and MDD groups as determined by the Mann-Whitney $U$-test. Employment status, marital status, presence of offspring, presence of a first-degree relative with a mood disorder, and presence of comorbid physical disease were significantly different as determined by the $\chi^{2}$ test. Cronbach's alpha coefficients for the TEMPS-A subscales in control and MDD subjects were depressive 0.78 , cyclothymic 0.86 , hyperthymic 0.79 , anxious 0.91 , and irritable 0.80 , indicating excellent or good internal consistencies.

\section{Stepwise multivariate logistic regression analysis using control/MDD as the dichotomous dependent variable}

The putative explanatory variables that showed significant differences between the control and MDD groups, as determined by the Mann-Whitney $U$-test or $\chi^{2}$ test in Table 1 , were further analyzed in stepwise multivariate logistic regression analysis. Table 2 shows the results of a stepwise multivariate logistic regression analysis in which the control/MDD was the dependent variable and employment status (yes $=1$, no $=0$ ), comorbid physical disease (yes $=1$, no $=0$ ), married (yes $=1$, no $=0$ ), presence of offspring (yes $=1$, no $=0$ ), having a first-degree relative with MDD (yes $=1$, no $=0$ ), duration of education (years), negative change score in the LES, depressive (high $=1$, low $=0$ ), cyclothymic (high $=1$, low $=0$ ), irritable (high $=1$, low $=0$ ), and anxious (high $=1$, low $=0$ ) temperament scores on the TEMPS-A; a neglect score and sexual scores of the CATS; and PHQ-9 summary scores were the independent variables. The subscale scores of TEMPS-A were divided into two categories, low or high, because the natural logarithm of the odds ratio was not linear with these variables. The total score on the CATS that had a significant difference (Table 1) was excluded from the stepwise multivariate logistic regression analysis because it had a high correlation with a neglect score on the CATS ( $P=0.92)$.

When entering these independent variables in a stepwise multivariate logistic regression analysis, the cyclothymic and anxious scores of TEMPS-A, comorbid physical disease, employment status, a neglect score on the CATS, and the PHQ-9 summary scores were significant predictors of MDD, whereas other variables were excluded from the model. The fit indexes of this model were as follows: $\chi^{2}=194.757$ $(P<0.001)$, Nagelkerke $R^{2}=0.707$, Hosmer-Lemeshow test $=0.316$, and predictive accuracy $=89.2 \%$. 
Table I Demographic and clinical characteristics and psychological measurement scores of healthy controls and patients with MDD

\begin{tabular}{|c|c|c|c|}
\hline Characteristics or measures & Control $(n=170)$ & MDD $(n=98)$ & $P$-value \\
\hline Age, years & $44.2 \pm 10.4$ & $45.6 \pm 10.6$ & 0.375 \\
\hline Sex (male:female) & 103:67 & $65: 33$ & 0.350 \\
\hline Education, years & $14.9 \pm 2.1$ & $14.1 \pm 2.4$ & 0.002 \\
\hline Employment status (employed:unemployed) & 139:30 & $67: 30$ & 0.013 \\
\hline Homemakers of unemployed persons & 28 & 14 & \\
\hline \multicolumn{4}{|l|}{ Marital status } \\
\hline Single & 23 & 26 & \\
\hline Married & 139 & 61 & \\
\hline Divorce & 7 & 8 & \\
\hline Widowed & 1 & 2 & \\
\hline Married (yes:no) & $|39: 3|$ & $61: 36$ & 0.001 \\
\hline Living alone (yes:no) & $33: 135$ & $18: 77$ & 0.891 \\
\hline Number of offspring & $1.47 \pm 1.13$ & $0.96 \pm 1.13$ & $<0.001$ \\
\hline Presence of offspring (yes:no) & $124: 46$ & $49: 46$ & $<0.001$ \\
\hline First-degree relative with mood disorder (yes:no) & $7: 163$ & $14: 80$ & 0.002 \\
\hline MDD & 7 & $12^{\mathrm{a}}$ & 0.009 \\
\hline Bipolar disorder & 0 & $3^{\mathrm{a}}$ & 0.044 \\
\hline Comorbid physical disease (yes:no) & $30: 140$ & 38:55 & $<0.001$ \\
\hline Comorbid psychiatric disorder (yes:no) & & $1 \mathrm{I}^{\mathrm{b}}: 84$ & \\
\hline Melancholic feature (yes:no) & & $23: 72$ & \\
\hline \multicolumn{4}{|l|}{ Number of previous depressive episode } \\
\hline 0 & & 47 & \\
\hline 1 & & 21 & \\
\hline $2-4$ & & 16 & \\
\hline$>4$ & & 12 & \\
\hline Illness duration, years & & $7.7 \pm 7.1$ & \\
\hline PHQ-9 score & $1.9 \pm 2.2$ & $9.6 \pm 6.7$ & $<0.001$ \\
\hline \multicolumn{4}{|l|}{ Number of psychopharmacologic treatments in use } \\
\hline Antidepressants (yes:no) (\%) & & $88: 10(90 \%)$ & \\
\hline Anxiolytics (yes:no) (\%) & & $76: 22(78 \%)$ & \\
\hline Atypical antipsychotics (yes:no) (\%) & & $26: 72(27 \%)$ & \\
\hline Mood stabilizer (yes:no) (\%) & & $24: 74(24 \%)$ & \\
\hline \multicolumn{4}{|l|}{ CATS (average score) } \\
\hline Sexual abuse & $0.02 \pm 0.09$ & $0.10 \pm 0.25$ & $<0.001$ \\
\hline Neglect & $0.42 \pm 0.48$ & $1.00 \pm 0.70$ & $<0.001$ \\
\hline Punishment & $1.42 \pm 0.60$ & $1.38 \pm 0.77$ & 0.454 \\
\hline Total score & $0.55 \pm 0.34$ & $0.87 \pm 0.52$ & $<0.001$ \\
\hline \multicolumn{4}{|l|}{ TEMPS-A (average score) } \\
\hline Depressive & $1.29 \pm 0.14$ & $1.48 \pm 0.21$ & $<0.001$ \\
\hline Cyclothymic & $1.09 \pm 0.10$ & $1.28 \pm 0.23$ & $<0.001$ \\
\hline Hyperthymic & $1.22 \pm 0.17$ & $1.18 \pm 0.17$ & 0.076 \\
\hline Anxious & $I . I I \pm 0.1 \mathrm{I}$ & $1.39 \pm 0.25$ & $<0.001$ \\
\hline Irritable & $1.07 \pm 0.10$ & $1.18 \pm 0.16$ & $<0.001$ \\
\hline \multicolumn{4}{|l|}{ LES (change score) } \\
\hline Negative & $1.62 \pm 4.04$ & $6.60 \pm 7.00$ & $<0.001$ \\
\hline Positive & $1.48 \pm 2.92$ & $1.84 \pm 3.38$ & 0.151 \\
\hline
\end{tabular}

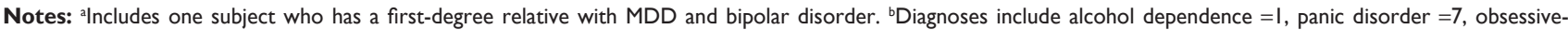
compulsive disorder $=2$, generalized anxiety disorder $=I$, and social anxiety disorder $=1$. Data represent the mean \pm SD or numbers. Owing to missing data, the sample size differs across the variables. The comparison of continuous data was analyzed with the Mann-Whitney $U$-test, and the comparison of categorical data was analyzed with a $\chi^{2}$ test and, when appropriate, with the Fisher's exact test.

Abbreviations: MDD, major depressive disorder; PHQ-9, Patient Health Questionnaire-9; CATS, Child Abuse and Trauma Scale; TEMPS-A, Temperament Evaluation of the Memphis, Pisa, Paris, and San Diego Autoquestionnaire; LES, Life Experiences Survey. 
Table 2 The results of a stepwise multivariate logistic regression model using control/MDD patients as the dependent variable

\begin{tabular}{llll}
\hline Variable & P-value & Odds ratio & $\mathbf{9 5 \%} \mathbf{~ C l}$ \\
\hline PHQ-9 & $<0.001$ & 1.43 & $1.25-1.63$ \\
Comorbid physical disease & 0.001 & 4.83 & $1.94-12.02$ \\
Cyclothymic score of TEMPS-A & 0.007 & 3.32 & $1.38-7.95$ \\
Anxious score of TEMPS-A & 0.010 & 3.02 & $1.30-7.02$ \\
Employment status & 0.030 & 0.33 & $0.12-0.90$ \\
Neglect score of CATS & 0.040 & 2.04 & $1.03-4.02$ \\
Presence of offspring & 0.083 & 0.46 & $0.19-1.11$ \\
\hline
\end{tabular}

Notes: Odds of MDD: values greater than I indicate that a higher score is associated with greater likelihood of MDD and values less than I indicate that a lower score is associated with a greater likelihood of being a control. Fourteen independent variables were analyzed: employment status (yes $=I$, no $=0$ ), comorbid physical disease (yes $=I$, no $=0$ ), married (yes $=I$, no $=0$ ), presence of offspring (yes $=I$, no $=0$ ), first-degree relative with MDD (yes $=1$, no $=0$ ), education years, negative change score of the LES, depressive (high $=1[>1.33]$, low $=0[\leqq 1.33]$ ), cyclothymic (high $[>1.14]=1$, low $[\leqq 1.14]=0$ ), irritable (high $[>1.00]=1$, low $[=1.00]=0$ ) and anxious (high $[>1.15]=1$, low $[\leqq 1.15]=0$ ) scores of the TEMPS-A, the neglect and sexual scores of the CATS, and the PHQ-9 summary score. $\mathrm{N}=170$ (Control) and $\mathrm{N}=98$ (MDD). Fit index of this model: $\chi^{2}=194.757(P<0.001)$. Nagelkerke $R^{2}=0.707$. Hosmer-Lemeshow test $=0.3 \mathrm{I}$. Predictive accuracy $=89.2 \%$.

Abbreviations: MDD, major depressive disorder; $\mathrm{Cl}$, confidence interval; PHQ-9, Patient Health Questionnaire-9; TEMPS-A, Temperament Evaluation of the Memphis, Pisa, Paris, and San Diego Autoquestionnaire; CATS, Child Abuse and Trauma Scale.

\section{Correlation between the CATS subscale scores and the temperament scores on the TEMPS-A in MDD patients}

As shown in Table 3, the four temperament scores (excluding the hyperthymic temperament score) of MDD patients were significantly and positively correlated with the neglect subscale scores. The punishment subscale score was significantly and positively correlated with the depressive temperament score. The sexual abuse subscale score was significantly and positively correlated with the depressive, cyclothymic, and anxious temperament scores.

Table 3 Correlation $(\rho)$ between the CATS subscales and the temperament scores of the TEMPS-A in patients with major depressive disorder

\begin{tabular}{lllllllll}
\hline & neg & pun & sex & dep & cyc & hyp & anx & irr \\
\hline neg & 1.00 & $0.47^{* *}$ & 0.18 & $0.38^{* *}$ & $0.35^{* *}$ & -0.04 & $0.37^{* *}$ & $0.35^{* *}$ \\
pun & & 1.00 & -0.10 & $0.28^{* *}$ & 0.15 & -0.12 & 0.12 & 0.18 \\
sex & & & 1.00 & $0.21^{*}$ & $0.21^{*}$ & -0.02 & $0.22^{*}$ & 0.03 \\
dep & & & & 1.00 & $0.42^{* *}$ & -0.04 & $0.63^{* *}$ & $0.50^{* *}$ \\
cyc & & & & 1.00 & 0.09 & $0.61^{* *}$ & $0.61^{* *}$ \\
hyp & & & & & 1.00 & -0.11 & -0.01 \\
anx & & & & & & 1.00 & $0.68^{* *}$ \\
irr & & & & & & & 1.00 \\
\hline
\end{tabular}

Notes: $\rho=$ Spearman's rank correlation coefficient. $* P<0.05$ and $* * P<0.01$.

Abbreviations: CATS, Child Abuse and Trauma Scale; TEMPS-A, Temperament Evaluation of the Memphis, Pisa, Paris, and San Diego Autoquestionnaire; neg, neglect subscale; pun, punishment subscale; sex, sexual abuse subscale; dep, depressive temperament; cyc, cyclothymic temperament; hyp, hyperthymic temperament; anx, anxious temperament; irr, irritable temperament.
Multiple regression analysis was performed to identify the independent predictors of each affective temperament on the TEMPS-A from the CATS subscales in MDD patients. Table 4 shows the results of the multiple regression analysis where each temperament score was the dependent factor and the neglect, punishment, and sexual abuse subscale scores on the CATS were the independent factors. The hyperthymic temperament score was not analyzed because Spearman's rank correlation coefficients showed no correlation between the hyperthymic temperament score and the three subscale scores of the CATS. Only the neglect subscale score was a significant predictor of the four temperament scores (Table 4).

\section{Analysis of the SEM using control/MDD as the dichotomous dependent variable}

To examine the association of all the variables, we built a structural equation model based on the results of the above correlation analysis and multivariate logistic regression analysis. The results of the path coefficients calculated by Mplus are shown in Figure 1.

Figure 1 shows the construction of the structural equation model, in which the standardized coefficients (with a maximum of 1 and a minimum of -1) are shown. Based on the demographic and clinical data and psychological measurement values (Table 1), the results of the multivariate logistic regression analysis (Table 2), the correlation between the CATS subscales and the temperament scores of the TEMPS-A (Table 3), and the multiple regression analysis of each temperament score of the TEMPS-A (Table 4), the observed variables constructed to the structural equation model were selected. Only the neglect subscale of the CATS was selected as the observed variable of childhood

Table 4 Correlation $(\rho)$ between the CATS subscales and the temperament scores of the TEMPS-A in patients with major depressive disorder

\begin{tabular}{|c|c|c|c|c|}
\hline \multirow{2}{*}{$\begin{array}{l}\text { Independent } \\
\text { factor }\end{array}$} & \multicolumn{4}{|c|}{ Dependent factor } \\
\hline & Depressive & Cyclothymic & Anxious & Irritable \\
\hline Neglect subscale & $0.26 *$ & $0.32 * *$ & $0.36 * *$ & $0.35 * *$ \\
\hline Punishment subscale & 0.17 & 0.01 & -0.04 & 0.08 \\
\hline Sexual abuse subscale & 0.18 & 0.15 & 0.15 & -0.03 \\
\hline ANOVA & $F=6.96 * * *$ & $F=5.27 * *$ & $F=5.99 * *$ & $F=4.4 I^{* *}$ \\
\hline Adjusted $R^{2}$ & 0.16 & 0.12 & 0.13 & 0.10 \\
\hline
\end{tabular}

Notes: Figures except the $F$-values and adjusted $R^{2}$ present beta values (standardized partial regression coefficients). Hyperthymic temperament was not analyzed because it was not correlated with any CATS subscale score (Table 3). $\rho=$ Spearman's rank correlation coefficient. $* P<0.05, * * P<0.01$, and $* * * P<0.001$.

Abbreviations: CATS, Child Abuse and Trauma Scale; TEMPS-A, Temperament Evaluation of the Memphis, Pisa, Paris, and San Diego Autoquestionnaire; ANOVA, analysis of variance. 


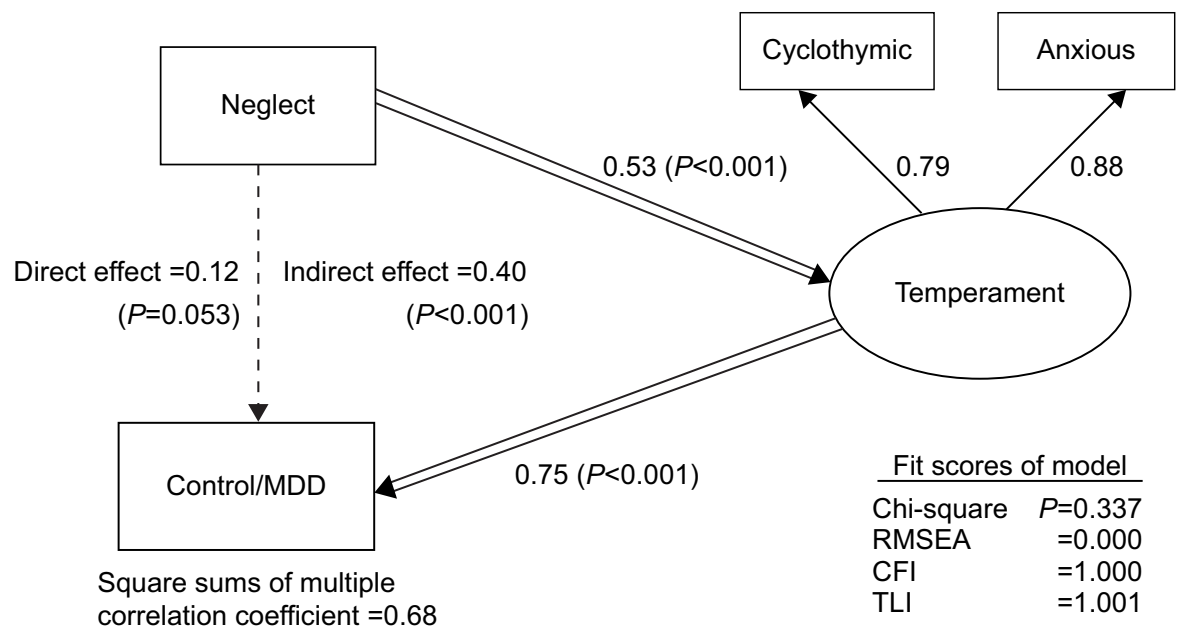

Figure I The results of the structural equation model for the 170 healthy control subjects and 98 MDD patients with neglect (CATS), affective temperaments (TEMPS-A), and control vs MDD.

Notes: Rectangles indicate the observed variables associated with the latent variable, which are shown as an oval. The arrows with double lines represent statistically significant paths, and the broken line shows the non-significant path. The numbers beside the arrows show the standardized path coefficients (minimum $-\mathrm{I}$, maximum I). The $P$-values reveal the levels of statistical significance. Indirect effects indicate the effect mediated by the other variables. A fit of the model is obtained as follows: the $\chi^{2}$ statistic $P=0.337$, RMSEA $=0.000, \mathrm{CFI}=1.000$, and $\mathrm{TLI}=1.00 \mathrm{I}$.

Abbreviations: MDD, major depressive disorder; CATS, Child Abuse and Trauma Scale; TEMPS-A, Temperament Evaluation of the Memphis, Pisa, Paris, and San Diego Autoquestionnaire; RMSEA, root mean square error of approximation; CFI, comparative fit index; TLI, Tucker-Lewis index.

abuse because the punishment and sexual subscales were not significant predictors of MDD (Table 2) or each temperament of the TEMPS-A (Table 4). Hyperthymic temperament was excluded from the observed variables of the latent variable temperament because hyperthymic temperament was neither different between the control and MDD groups (Table 1) nor correlated with any subscales of the CATS (Table 3). Furthermore, depressive and irritable temperaments were excluded from the observed variables of the latent variable temperament because the multiple fit criteria were not enough in the SEM and these two temperaments were not significant predictors of MDD (Table 2).

A good fit of the model was obtained as follows: the $\chi^{2}$ statistic $P=0.337$, RMSEA $=0.000, \mathrm{CFI}=1.000$, and $\mathrm{TLI}=1.001$. Only the path coefficient of neglect to control/ MDD (0.12) was not significant $(P=0.053)$. The other path coefficients were substantially significant $(P<0.001)$.

Consistent with the results of the multiple regression analysis (Table 4), two temperament scores on the TEMPS-A were significantly predicted by the subscale neglect of the CATS in the SEM (Figure 1). Having MDD vs being a control was significantly predicted by two temperament scores on the TEMPS-A. The effect of the neglect score on the prediction of having MDD vs being a control was indirect and mediated by the effect of the neglect score on the two temperament scores in the TEMPS-A (indirect path coefficient $=0.40, P<0.001)$.

\section{Demographic and clinical characteristics and the PHQ-9, CATS, TEMPS-A, and LES scores of healthy controls and the remission and treatment-resistant groups of MDD patients}

The demographic and clinical characteristics and the PHQ-9, CATS, TEMPS, and LES scores of 170 healthy controls and the 55 remission and 22 treatment-resistant MDD patients are presented in Table 5. The STAI-Y, HDRS, and YMRS results are investigated only in the MDD patients (Table 5).

The duration of education; the number of married subjects; the employment status; the number of offspring; the presence of offspring; the presence of a first-degree relative with a mood disorder; the presence of a first-degree relative with MDD; the presence of comorbid physical disease; the PHQ-9 summary scores; the sexual, neglect, and total scores of the CATS; the depressive, cyclothymic, anxious, and irritable temperament scores of the TEMPS; and the negative change score of the LES were significantly different between these three groups as determined by the Kruskal-Wallis test.

The remission group had significantly shorter durations of education than the healthy controls, as determined by the Steel-Dwass post hoc analysis. The remission group had significantly higher scores or higher frequencies of the presence of comorbid physical disease; the PHQ-9 summary scores; the sexual, neglect, and total scores of the 
Table 5 Demographic and clinical characteristics and psychological measurement scores of healthy controls and patients with MDD (remission and treatment-resistant groups)

\begin{tabular}{|c|c|c|c|c|c|}
\hline \multirow[t]{2}{*}{ Characteristics or measures } & \multirow{2}{*}{$\begin{array}{l}\text { Control } \\
(n=\mid 70)\end{array}$} & \multicolumn{2}{|l|}{ MDD } & \multirow{2}{*}{$\begin{array}{l}H \text {-value } \\
\text { (Kruskal- } \\
\text { Wallis test) }\end{array}$} & \multirow[t]{2}{*}{ P-value } \\
\hline & & $\begin{array}{l}\text { Remission } \\
\text { group }(n=55)\end{array}$ & $\begin{array}{l}\text { Treatment-resistant } \\
\text { group }(n=22)\end{array}$ & & \\
\hline Age, years & $44.2 \pm 10.4$ & $47.9 \pm 11.9$ & $42.9 \pm 6.9$ & 4.27 & 0.118 \\
\hline Sex (male:female) & 103:67 & $35: 20$ & $16: 6$ & 1.27 & 0.531 \\
\hline Education, years & $14.9 \pm 2.1$ & $13.8 \pm 2.4 * *$ & $14.7 \pm 2.5$ & 11.61 & 0.003 \\
\hline Employment status (employed:unemployed) & $139: 30$ & $37: 17$ & $14: 8$ & 7.09 & 0.029 \\
\hline Homemakers of unemployed persons & 28 & 11 & I & & \\
\hline \multicolumn{6}{|l|}{ Marital status } \\
\hline Single & 23 & 10 & 8 & & \\
\hline Married & 139 & 40 & 10 & & \\
\hline Divorce & 7 & 4 & 3 & & \\
\hline Widowed & I & I & I & & \\
\hline Married (yes:no) & $|39: 3|$ & $40: 15$ & $10: 12^{* *}$ & 14.80 & $<0.001$ \\
\hline Living alone (yes:no) & $33: 135$ & $5: 48$ & $6: 16$ & 4.19 & 0.123 \\
\hline Number of offspring & $1.47 \pm 1.13$ & $1.25 \pm 1.07$ & $0.45 \pm 0.80 * * \ldots$ & 18.13 & $<0.001$ \\
\hline Presence of offspring (yes:no) & $124: 46$ & $35: 18$ & $7: 15^{* *, \#}$ & 14.80 & $<0.001$ \\
\hline First-degree relative with mood disorder (yes:no) & $7: 163$ & $6: 47$ & $5: 17 * *$ & 11.05 & 0.004 \\
\hline MDD & 7 & 5 & $5^{\mathrm{a}, * *}$ & 11.43 & 0.003 \\
\hline Bipolar disorder & 0 & 1 & $\mathrm{I}^{\mathrm{a}}$ & 5.88 & 0.052 \\
\hline Comorbid physical disease (yes:no) & $30: 140$ & $22: 30 * *$ & $8: 14$ & $|4.8|$ & $<0.001$ \\
\hline Comorbid psychiatric disorder (yes:no) & & $7^{\mathrm{b}: 48}$ & $3^{c}: 19$ & Fisher & 0.589 \\
\hline Melancholic feature (yes:no) & & $7: 45$ & $12: 10$ & Chi-square & $<0.001$ \\
\hline Recurrent major depression (yes:no) & & $24: 31$ & $16: 6$ & Chi-square & 0.021 \\
\hline \multicolumn{6}{|l|}{ Number of previous depressive episode } \\
\hline 0 & & 30 & 8 & & \\
\hline 1 & & 8 & 5 & & \\
\hline $2-4$ & & 115 & 35 & & \\
\hline$>4$ & & 5 & 5 & & \\
\hline Illness duration, years & & $7.9 \pm 8.2$ & $8.5 \pm 5.9$ & $U$-test & 0.301 \\
\hline PHQ-9 (summary score) & $1.9 \pm 2.2$ & $6.5 \pm 4.7 * *$ & $16.5 \pm 7.5^{* *}$ & 89.55 & $<0.001$ \\
\hline HDRS (total score) & & $4.6 \pm 4.1$ & $16.1 \pm 8.9^{\#}$ & $U$-test & $<0.001$ \\
\hline YMRS (total score) & & $0.4 \pm 1.1$ & $1.7 \pm 3.4^{\#}$ & $U$-test & 0.026 \\
\hline \multicolumn{6}{|l|}{ STAI-Y (total score) } \\
\hline State & & $41.9 \pm 12.5$ & $52.7 \pm 16.4^{\#}$ & $U$-test & 0.001 \\
\hline Trait & & $45.9 \pm 13.4$ & $57.9 \pm 18.3^{\#}$ & $U$-test & $<0.001$ \\
\hline \multicolumn{6}{|l|}{ CATS (average score) } \\
\hline Sexual abuse & $0.02 \pm 0.09$ & $0.09 \pm 0.24 * *$ & $0.17 \pm 0.35 * *$ & 15.86 & $<0.001$ \\
\hline Neglect & $0.42 \pm 0.48$ & $0.79 \pm 0.57 * *$ & $1.55 \pm 0.69 * * \ldots$ & 54.88 & $<0.001$ \\
\hline Punishment & $1.42 \pm 0.60$ & $1.36 \pm 0.66$ & $1.48 \pm 0.79$ & 0.83 & 0.661 \\
\hline Total & $0.55 \pm 0.34$ & $0.74 \pm 0.4 I^{* *}$ & $1.22 \pm 0.5 \mathrm{I} * * \ldots \#$ & 38.08 & $<0.001$ \\
\hline \multicolumn{6}{|l|}{ TEMPS-A (average score) } \\
\hline Depressive & $1.29 \pm 0.14$ & $1.42 \pm 0.21 * *$ & $1.62 \pm 0.16 * *$ & 59.24 & $<0.001$ \\
\hline Cyclothymic & $1.09 \pm 0.10$ & $1.24 \pm 0.20 * *$ & $1.37 \pm 0.24 * *, \#$ & 60.61 & $<0.001$ \\
\hline Hyperthymic & $1.22 \pm 0.17$ & $1.17 \pm 0.15$ & $1.20 \pm 0.16$ & 3.08 & 0.215 \\
\hline Anxious & $I . I I \pm 0.1 \mathrm{I}$ & $1.35 \pm 0.24 * *$ & $1.52 \pm 0.24 * *, \#$ & 77.31 & $<0.001$ \\
\hline Irritable & $1.07 \pm 0.10$ & $1.15 \pm 0.15 * *$ & $1.25 \pm 0.17 * *, \#$ & 36.84 & $<0.001$ \\
\hline \multicolumn{6}{|l|}{ LES (total score) } \\
\hline Negative & $1.62 \pm 4.04$ & $4.62 \pm 5.14 * *$ & $10.09 \pm 8.54 * *, \#$ & 55.32 & $<0.001$ \\
\hline Positive & $1.48 \pm 2.92$ & $1.69 \pm 2.44$ & $2.82 \pm 5.58$ & 2.95 & 0.229 \\
\hline
\end{tabular}

Notes: ancludes one subject who has a first-degree relative with a MDD and bipolar disorder. ${ }^{b}$ Diagnoses include alcohol dependence $=1$, panic disorder $=5$, generalized anxiety disorder $=\mathrm{I}$, and social anxiety disorder $=\mathrm{I}$. ' Diagnoses include panic disorder $=2$ and panic disorder + obsessive-compulsive disorder $=I$. $* * P<0.0 \mathrm{I}$ vs controls, ${ }^{\#} P<0.05$ vs the remission group, and ${ }^{\# P} P 0.01$ vs the remission group. The comparison of the three groups was analyzed by the Kruskal-Wallis test followed by the Steel-Dwass test, and the comparison of the two groups was analyzed by the $U$-test. Data represent the mean \pm SD or numbers. Owing to missing data, the sample size differs across the variables.

Abbreviations: MDD, major depressive disorder; PHQ-9, Patient Health Questionnaire-9; HDRS, Hamilton Depression Rating Scale; YMRS, Young Mania Rating Scale; STAI-Y, Spielberger State-Trait Anxiety Inventory, Form Y; CATS, Child Abuse and Trauma Scale; TEMPS-A, Temperament Evaluation of the Memphis, Pisa, Paris, and San Diego Autoquestionnaire; LES, Life Experiences Survey. 
CATS; the depressive, cyclothymic, anxious, and irritable temperament scores of the TEMPS; and the negative change score of the LES compared to the healthy controls. The treatment-resistant group had significantly lower scores or lower frequencies of the number of married subjects, the number of offspring, and the presence of offspring compared to the healthy controls, as determined by the Steel-Dwass post hoc analysis. The treatment-resistant group had significantly higher scores or higher frequencies of the presence of a first-degree relative with a mood disorder; the presence of a first-degree relative with MDD; the PHQ-9 summary scores; the sexual, neglect, and total scores of the CATS; the depressive, cyclothymic, anxious, and irritable temperament scores of the TEMPS; and the negative change score of the LES than healthy controls.

The treatment-resistant group had significantly lower scores or lower frequencies of the number of offspring and the presence of offspring than the remission group, as determined by the Steel-Dwass post hoc analysis. The treatment-resistant group had significantly higher scores or higher frequencies on the PHQ-9 summary scores; the neglect and total scores of the CATS; the depressive, cyclothymic, irritable, and anxious temperament scores of the TEMPS; the negative change score of the LES; the HDRS scores; the YMRS scores; and the state and trait anxiety scores of the STAI-Y than the remission group as determined by the Steel-Dwass post hoc analysis or the Mann-Whitney $U$-test.

\section{Multivariate logistic regression analysis of remission vs treatment-resistance in patients with MDD}

To confirm whether the CATS and TEMPS-A scores are predictors of remission vs treatment-resistance in patients with MDD independently of depressive symptoms (PHQ-9 summary scores), multivariate logistic regression analyses were performed where remission vs treatment-resistance in patients with MDD was the dependent variable (data not shown). Neglect $(P=0.020$, odds ratio $=4.10)$ and the total scores $(P=0.041$, odds ratio $=5.15)$ of the CATS were independent predictors of remission vs treatment-resistance in patients with MDD when a PHQ-9 summary score and each of these two subscales of the CATS were the two independent variables. However, the sexual and punishment scores of the CATS, all the subscale scores of the TEMPS-A, and the negative change scores of the LES were not significantly independent predictors of remission vs treatment-resistance in patients with MDD when the PHQ-9 score and each of these scores were included as the two independent variables.

\section{Discussion}

This study is the first report showing that childhood abuse indirectly predicted MDD through the affective temperaments measured by the TEMPS-A in the SEM comparing healthy controls and patients with MDD. Two temperaments cyclothymic and anxious - directly predicted the diagnosis of MDD. The validity of this result of the SEM was supported by the results of the multivariate logistic regression analysis based on the clinical and demographic characteristics and the questionnaire data that compared the control and MDD groups as follows: a stepwise multivariate logistic analysis showed that three factors - neglect, cyclothymic, and anxious temperaments - predicted the diagnosis of MDD independently of the severity of depressive symptoms. These factors were statistically significant predictors or factors with high path coefficients in the SEM. These results are consistent with the results of our recent study in a nonclinical general adult population, in which childhood abuse indirectly increased the severity of depressive symptoms through increased affective temperaments, and the affective temperaments directly increased the severity of depressive symptoms in the SEM. ${ }^{9}$

In our previous study of a nonclinical general adult population, ${ }^{9}$ a neglect score on the CATS was a significant predictor of depressive, cyclothymic, irritable, and anxious temperaments in the multiple regression analysis. Clinically, Pompili et $\mathrm{al}^{34}$ reported that psychiatric inpatients with a history of childhood abuse had a higher incidence of the irritable temperament trait than the non-abused patients. However, no study has examined the effect of childhood abuse on the TEMPS-A in MDD patients. In the multiple regression analysis of this study, only neglect among the childhood abuse subscales significantly predicted high scores of depressive, cyclothymic, irritable, and anxious temperaments in MDD patients, which is consistent with our previous results in a nonclinical general adult population. ${ }^{9}$ Moreover, the result of the SEM indicates that a history of neglect indirectly predicts the diagnosis of MDD through the affective temperaments, especially the cyclothymic and anxious temperaments, measured by the TEMPS-A.

On the other hand, hyperthymic temperament was not significantly associated with childhood abuse in this study. Rovai et $\mathrm{al}^{35}$ noted that there is a gap between hyperthymic temperament, which must be considered the most functional and desirable, and cyclothymic, depressive, irritable and anxious temperaments, which are closer to mood, anxiety, and substance use disorders, and imply difficulty in adapting emotionally and behaviorally to somatic diseases and 
life stressors. Our study suggests that a history of childhood abuse is implicated in the gap between hyperthymic temperament and the other four temperaments in adulthood.

Partly consistent with our present research, a previous study reported that the depressive, cyclothymic, and anxious temperament scores of MDD patients were higher than those of healthy controls. ${ }^{28}$ In the present study, four temperaments depressive, cyclothymic, irritable, and anxious - measured by the TEMPS-A in MDD patients were higher than those in the controls independently of the depressive symptoms in univariate analyses: the remission MDD group also had significantly higher scores. However, in a multivariate logistic regression analysis, only the cyclothymic and anxious temperaments were significant predictors of MDD. This finding was also supported by higher path coefficients from the latent variable temperament to cyclothymic and anxious temperaments in the SEM. These results agree with the results of our previous study in a nonclinical general adult population that cyclothymic and anxious temperaments were significant predictors of depressive symptoms in a multiple regression analysis. ${ }^{9}$ Our study suggests that high cyclothymic and anxious temperament scores are important factors for predicting the onset of MDD.

The affective temperaments measured by the TEMPS-A are considered antecedents or subsyndromal manifestations of mood disorders. As discussed in the above paragraph, the cyclothymic and anxious temperament scores of MDD patients are higher than those of healthy controls in the present and previous studies. ${ }^{28}$ In particular, cyclothymic temperament is more evident in bipolar disorders, ${ }^{36,37}$ indicating biological implications. In contrast, anxious temperament has an inverse relationship with resilience both in depressive and healthy individuals ${ }^{38}$ and is a robust predictor of most psychiatric disorders, especially within the anxiety and depressive clusters. ${ }^{39}$ On the other hand, childhood abuse is also a major environmental factor that predicts MDD. ${ }^{3-6,12}$ However, the association between childhood abuse and affective temperament at the onset of MDD has not been clarified. Our previous study in a nonclinical general adult population suggested the possibility that childhood abuse causes depression through increasing affective temperaments. ${ }^{9}$ The present study supports this hypothesis and suggests that childhood abuse, especially neglect, increases cyclothymic and anxious temperaments, which, in turn, might affect the onset or clinical outcomes of MDD.

This study revealed that the total and neglect scores on the CATS in the stage 2 MDD-TRD group were significantly higher than those in the remission group. Whereas a meta-analysis ${ }^{13}$ recently showed that childhood maltreatment was associated with an elevated risk of lack of response or remission during treatment for depression, the present study is the first report in which childhood abuse is associated with the stage 2 MDD-TRD. The significant difference of the total and neglect scores on the CATS between the stage 2 MDD-TRD and remission groups were not diminished when the PHQ-9 summary scores were included as the independent factor in a multivariate logistic regression analysis. Therefore, the neglect and total scores on the CATS were associated with the stage 2 MDD-TRD independently of the severity of the depressive symptoms.

In this study, the stage 2 MDD-TRD group had higher scores of depressive, cyclothymic, anxious, and irritable temperament and higher negative change scores on the LES compared with the remission group. Although there is a possibility that affective temperament plays a role in mediating the influence of childhood abuse on the stage 2 MDD-TRD group, as seen in the SEM of healthy controls and patients with MDD (Figure 1), the multivariate analysis and SEM of stage 2 MDD-TRD could not be performed because of an insufficient sample size. Additionally, the significant differences of the affective temperament and the negative change score on the LES between these groups were not independent of depressive symptoms in multivariate logistic regression analyses. Therefore, depressive symptoms may be a confounding factor for the TEMPS-A and LES data of the remission and stage 2 MDD-TRD groups, which constitutes a limitation of this study. Further studies with a larger sample size of patients with TRD will be necessary.

Another limitation of this study is that childhood abuse was estimated retrospectively and affective temperament was not evaluated before the onset of MDD. To confirm the influence of childhood abuse and temperaments on the onset of MDD, a prospective longitudinal study of a representative birth cohort will be necessary. Additionally, the CATS, the scale used to measure childhood abuse in this study, is a questionnaire in which patients recall the experiences in childhood, whereby psychological symptoms such as depressive symptoms on a test day may influence the recollection of a traumatic event in childhood. However, because the neglect score of the CATS predicted the diagnosis of MDD in the multivariate logistic analysis, including the PHQ summary score as an independent variable, the influence of depressive symptoms on the neglect score of the CATS in this study may not be significant.

The SEM of this study showed that childhood abuse, especially neglect, increased affective temperament, which, in turn, predicted MDD. These results suggest the important role of affective temperament as a mediator in the 
effect of childhood abuse on MDD. Whereas this study also suggests that a history of neglect may be involved in treatment-resistance in patients with MDD, further studies using multivariate analysis will be needed to confirm these relationships in a larger sample size.

\section{Conclusion}

This study suggests that childhood abuse, especially neglect, indirectly predicted the diagnosis of MDD through increased affective temperaments. The important role as a mediator of affective temperaments in the effect of childhood abuse on MDD was suggested.

\section{Acknowledgments}

This study was partly supported by the program Integrated Research on Neuropsychiatric Disorders conducted under the Strategic Research Program for Brain Sciences by the Ministry of Education, Culture, Sports, Science, and Technology of Japan, a research grant 24-2 for nervous and mental disorders from the Ministry of Health, Labor and Welfare, and a grant from the Interdisciplinary Project for Psychosomatological Research in Hokkaido University. We thank Miss Satoko Fukushima and Rira Yamashita for managing the schedule of data collection and entering data to a database.

\section{Author contributions}

Dr Toda and Dr Inoue designed the study and wrote the protocol and analyzed data. Dr Toda, Dr Inoue, Dr Tsunoda, Dr Tanichi, Dr Tanaka, Dr Hashimoto, Dr Nakato, Dr Nakagawa, Dr Kitaichi, Dr Mitsui, and Ms Nakai collected data. All other authors checked the protocol and discussion. All authors contributed to and have approved the final manuscript. All authors contributed toward data analysis, drafting and critically revising the paper and agree to be accountable for all aspects of the work.

\section{Disclosure}

The authors report no financial or other relationship that is relevant to the subject of this article. HT has received honoraria from GlaxoSmithKline, Eli Lilly, Mitsubishi Tanabe Pharma, Otsuka Pharmaceutical, Meiji Seika Pharma, and Yoshitomi Pharmaceutical. TI has received honoraria from GlaxoSmithKline, Pfeizer, Astellas Pharma, Eli Lilly, Mitsubishi Tanabe Pharma, Mochida Pharmaceutical, Otsuka Pharmaceutical, Meiji Seika Pharma, Asahi Kasei Pharma, Shionogi, Janssen Pharmaceuticals, Takeda Pharmaceutical, and Yoshitomi Pharmaceutical; TI has received research/ grant support from Otsuka Pharmaceutical; and is a member of the advisory board of GlaxoSmithKline, Eli Lilly, Mochida Pharmaceutical, and Mitsubishi Tanabe Pharma. SN has received honoraria from GlaxoSmithKline, Eisai, Pfeizer, Daiichi Sankyo, Meiji Seika Pharma, Ono Pharmaceutical, and Eli Lilly and has received research/grant support from Pfeizer, Eli Lilly, Eisai, and Ono Pharmaceutical. IK has received honoraria from Eli Lilly, has received research/ grant support from Takeda Pharmaceutical, Astellas Pharma, and Dainippon Sumitomo Pharma, and is a member of the advisory board of Dainippon Sumitomo Pharma and Tanabe Mitsubishi Pharma. YK has received honoraria from Otsuka Pharmaceutical and Meiji Seika Pharma. The other authors declare that they have no actual or potential conflicts of interest.

\section{References}

1. Caspi A, Hariri AR, Holmes A, Uher R, Moffitt TE. Genetic sensitivity to the environment: the case of the serotonin transporter gene and its implications for studying complex diseases and traits. Am J Psychiatry. 2010;167(5):509-527.

2. Kendler KS, Neale MC, Kessler RC, Heath AC, Eaves LJ. A longitudinal twin study of 1-year prevalence of major depression in women. Arch Gen Psychiatry. 1993;50(11):843-852.

3. Kessler RC, Magee WJ. Childhood adversities and adult depression: basic patterns of association in a US national survey. Psychol Med. 1993;23(3):679-690.

4. Weich S, Patterson J, Shaw R, Stewart-Brown S. Family relationships in childhood and common psychiatric disorders in later life: systematic review of prospective studies. Br J Psychiatry. 2009; 194(5):392-398.

5. Wise LA, Zierler S, Krieger N, Harlow BL. Adult onset of major depressive disorder in relation to early life violent victimisation: a case-control study. Lancet. 2001;358(9285):881-887.

6. Caspi A, Sugden K, Moffitt TE, et al. Influence of life stress on depression: moderation by a polymorphism in the 5-HTT gene. Science. 2003;301(5631):386-389.

7. Kendler KS, Kuhn J, Prescott CA. The interrelationship of neuroticism, sex, and stressful life events in the prediction of episodes of major depression. Am J Psychiatry. 2004;161(4):631-636.

8. Gonda X, Fountoulakis KN, Juhasz G, et al. Association of the s allele of the 5-HTTLPR with neuroticism-related traits and temperaments in a psychiatrically healthy population. Eur Arch Psychiatry Clin Neurosci. 2009;259(2):106-113.

9. Nakai Y, Inoue T, Toda H, et al. The influence of childhood abuse, adult stressful life events and temperaments on depressive symptoms in the nonclinical general adult population. J Affect Disord. 2014; 158:101-107.

10. Bienvenu OJ, Davydow DS, Kendler KS. Psychiatric 'diseases' versus behavioral disorders and degree of genetic influence. Psychol Med.2011; 41(1):33-40.

11. Kendler KS, Karkowski LM, Prescott CA. Causal relationship between stressful life events and the onset of major depression. Am J Psychiatry. 1999; 156(6):837-841.

12. Heim C, Newport DJ, Mletzko T, Miller AH, Nemeroff CB. The link between childhood trauma and depression: insights from HPA axis studies in humans. Psychoneuroendocrinology. 2008;33(6):693-710.

13. Nanni V, Uher R, Danese A. Childhood maltreatment predicts unfavorable course of illness and treatment outcome in depression: a metaanalysis. Am J Psychiatry. 2012;169(2):141-151.

14. Thase ME, Rush AJ. Treatment-Resistant Depression. New York: Raven Press; 1995. 
15. Thase ME, Rush AJ. When at first you don't succeed: sequential strategies for antidepressant nonresponders. J Clin Psychiatry. 1997;58(suppl 13): 23-29.

16. Inoue $\mathrm{T}$, Nakagawa $\mathrm{S}$, Kitaichi $\mathrm{Y}$, et al. Long-term outcome of antidepressant-refractory depression: the relevance of unrecognized bipolarity. J Affect Disord. 2006;95(1-3):61-67.

17. Souery D, Oswald P, Massat I, et al; Group for the Study of Resistant Depression. Clinical factors associated with treatment resistance in major depressive disorder: results from a European multicenter study. J Clin Psychiatry. 2007;68(7):1062-1070.

18. Takahashi M, Shirayama Y, Muneoka K, Suzuki M, Sato K, Hashimoto K. Personality traits as risk factors for treatment-resistant depression. PLoS One. 2013;8(5):e63756.

19. Akiskal HS, Akiskal KK, Haykal RF, Manning JS, Connor PD. TEMPS-A: progress towards validation of a self-rated clinical version of the temperament evaluation of the Memphis, Pisa, Paris, and San Diego Autoquestionnaire. J Affect Disord. 2005;85(1-2):3-16.

20. Sanders B, Becker-Lausen E. The measurement of psychological maltreatment: early data on the Child Abuse and Trauma Scale. Child Abuse Negl. 1995;19(3):315-323.

21. Sarason IG, Johnson JH, Siegel JM. Assessing the impact of life changes: development of the Life Experiences Survey. J Consult Clin Psychol. 1978;46(5):932-946.

22. Amorim P, Lecrubier Y, Weiller E, Hergueta T, Sheehan D. The Mini International Neuropsychiatric Interview (MINI). A short diagnostic structured interview: reliability and validity according to the CIDI. Eur Psychiatry. 1997;12(5):224-231.

23. Spitzer RL, Kroenke K, Williams JB. Validation and utility of a selfreport version of PRIME-MD: the PHQ primary care study. Primary Care Evaluation of Mental Disorders. Patient Health Questionnaire. JAMA. 1999;282(18):1737-1744.

24. American Psychiatric Association. Practice Guidelines for the Treatment of Psychiatric Disorders. Compendium 2000. Washington, DC: American Psychiatric Assoiation; 2000.

25. Young RC, Biggs JT, Ziegler VE, Meyer DA. A rating scale for mania: reliability, validity and sensitivity. Br J Psychiatry. 1978;133: $429-435$.

26. Williams JB. A structured interview guide for the Hamilton Depression Rating Scale. Arch Gen Psychiatry. 1988;45(8):742-747.

27. Muramatsu K, Miyaoka H, Kamijima K, et al. The patient health questionnaire, Japanese version: validity according to the mini-international neuropsychiatric interview-plus. Psychol Rep. 2007;101(3 pt 1): 952-960.
28. Matsumoto S, Akiyama T, Tsuda H, et al. Reliability and validity of TEMPS-A in a Japanese non-clinical population: application to unipolar and bipolar depressives. J Affect Disord. 2005;85(1-2):85-92.

29. Tanabe H, Ozawa S, Goto K. Psychometric properties of the Japanese version of the Child Abuse and Trauma Scale (CATS). The 9th Annual Meeting of the Japanese Society for Traumatic Stress Studies (in Japanese); 2010.

30. Spielberger CD. Manual for the State-Trait Anxiety Inventory: STAI (Form Y). Palo Alto, CA: Consulting Psychologists Press; 1983.

31. Hidano T, Fukuhara M, Iwawaki S, Soga S, Spielberger CD. State-Trait Anxiety Inventory-Form JYZ. Tokyo, Japan: Jitsumu Kyoiku Shuppan; 2000

32. Schermelleh-Engel K, Moosbrugger H, Müller H. Evaluating the fit of structural equation models: tests of significance and descriptive goodnessof-fit measures. Methods Psychol Res Online. 2003;8(2):23-74.

33. Kanda Y. Investigation of the freely available easy-to-use software 'EZR' for medical statistics. Bone Marrow Transplant. 2013;48(3): 452-458.

34. Pompili M, Iliceto $\mathrm{P}$, Innamorati $\mathrm{M}$, et al. Suicide risk and personality traits in physically and/or sexually abused acute psychiatric inpatients: a preliminary study. Psychol Rep. 2009;105(2):554-568.

35. Rovai L, Maremmani AG, Rugani F, et al. Do Akiskal \& Mallya's affective temperaments belong to the domain of pathology or to that of normality? Eur Rev Med Pharmacol Sci. 2013;17(15):2065-2079.

36. Mendlowicz MV, Jean-Louis G, Kelsoe JR, Akiskal HS. A comparison of recovered bipolar patients, healthy relatives of bipolar probands, and normal controls using the short TEMPS-A. J Affect Disord. 2005; 85(1-2):147-151.

37. Mendlowicz MV, Akiskal HS, Kelsoe JR, Rapaport MH, Jean-Louis G, Gillin JC. Temperament in the clinical differentiation of depressed bipolar and unipolar major depressive patients. J Affect Disord. 2005;84(2-3):219-223.

38. Kesebir S, Gundogar D, Kucuksubasi Y, Tatlidil Yaylaci E. The relation between affective temperament and resilience in depression: a controlled study. J Affect Disord. 2013;148(2-3):352-356.

39. Karam EG, Salamoun MM, Yeretzian JS, et al. The role of anxious and hyperthymic temperaments in mental disorders: a national epidemiologic study. World Psychiatry. 2010;9(2):103-110.
Neuropsychiatric Disease and Treatment

\section{Publish your work in this journal}

Neuropsychiatric Disease and Treatment is an international, peerreviewed journal of clinical therapeutics and pharmacology focusing on concise rapid reporting of clinical or pre-clinical studies on a range of neuropsychiatric and neurological disorders. This journal is indexed on PubMed Central, the 'PsycINFO' database and CAS,

\section{Dovepress}

and is the official journal of The International Neuropsychiatric Association (INA). The manuscript management system is completely online and includes a very quick and fair peer-review system, which is all easy to use. Visit http://www.dovepress.com/testimonials.php to read real quotes from published authors. 